\title{
A Novel Bipartite Mode of Binding of $M$. smegmatis Topoisomerase I to its Recognition Sequence
}

\author{
Devanjan Sikder ${ }^{1}$ and Valakunja Nagaraja ${ }^{1,2 *}$
}

\author{
${ }^{1}$ Department of Microbiology \\ and Cell Biology, Indian \\ Institute of Science, Bangalore \\ 560 012, India \\ ${ }^{2}$ Jawaharlal Nehru Centre for \\ Advanced Scientific Research \\ Bangalore 560 054, India
}

\begin{abstract}
We have investigated interaction of Mycobacterium smegmatis topoisomerase I at its specific recognition sequence. DNase I footprinting demonstrates a large region of protection on both the scissile and non-scissile strands of DNA. Methylation protection and interference analyses reveal base-specific contacts within the recognition sequence. Missing contact analyses reveal additional interactions with the residues in both single and double-stranded DNA, and hence underline the role for the functional groups associated with those bases. These interactions are supplemented by phosphate contacts in the scissile strand. Conformation specific probes reveal protein-induced structural distortion of the DNA helix at the T-A-T-A sequence $11 \mathrm{bp}$ upstream to the recognition sequence. Based on these footprinting analyses that define parameters of topoisomerase I-DNA interactions, a model of topoisomerase I binding to its substrate is presented. Within the large protected region of $30 \mathrm{bp}$, the enzyme makes direct contact at two locations in the scissile strand, one around the cleavage site and the other 8-12 bases upstream. Thus the enzyme makes asymmetric recognition of DNA and could carry out DNA relaxation by either of the two proposed mechanisms: enzyme bridged and restricted rotation.
\end{abstract}

Keywords: footprinting; mycobacteria; Sequence specificity; Topoisomerase I type I enzymes are further categorized into two groups, IA and IB, based on the biochemical properties and the type of reaction intermediate. Members of type IA form a $5^{\prime}$ phospho-tyrosine adduct during the first trans-esterification reaction, and generally require a single-stranded region in the DNA and magnesium as a cofactor in a reaction restricted to relaxation of the negatively supercoiled DNA. Examples include Escherichia coli topoisomerase I and topoisomerase III, Bacillus subtilis topoisomerase I and reverse gyrase. ${ }^{4,5}$ Type IB enzymes, in contrast, form a covalent contact with the $3^{\prime}$ residue during the first catalytic step of trans-esterification. Members of this subclass utilize duplex DNA as substrate and are able to act on both positively and negatively supercoiled substrates in a metal ion-independent DNA relaxation reaction. Examples of this group include nuclear topoisomerase I found in all eukaryotic cells, topoisomerase $\mathrm{V}$, and the topoisomerase I encoded by the vaccinia and other cytoplasmic viruses. ${ }^{4}$

To gain insight into the function and reaction mechanism, attempts have been made to study the interaction of topoisomerase with substrate DNA. $^{6-11}$ The data obtained from various sys-
Abbreviations used: STS, strong topoisomerase site; SS, scissile strand.

E-mail address of the corresponding author: vraj@mcbl.iisc.ernet.in 
tems suggest that the sequence specificity of topoisomerases is not very stringent although they appear to prefer certain sequences to others., $72-18$ Topoisomerase I from vaccinia virus is perhaps the only exception, which is distinctive in its site specificity. ${ }^{8}$ Detailed studies have been carried out on the site specific interaction of vaccinia topoisomerase I with DNA and as a result the viral protein has emerged as the model for understanding the reaction mechanism of type IB topoisomerases. ${ }^{19}$ The biochemical knowledge on the enzyme was complemented by the structural information of the viral topoisomerase I. ${ }^{20,21}$ These studies have led to the proposal of free-rotation model of relaxation for the enzyme. ${ }^{22}$ Meanwhile, mechanistic studies with human topoisomerase I-DNA complexes provided insights into the structural features important for the interaction of the latter enzyme, leading to a different scheme of DNA relaxation. $^{23,24}$

Three-dimensional structure of the truncated E. coli topoisomerase $\mathrm{I}^{9}$ provided data about the domains of the protein, and suggested a probable relaxation mechanism. Based on these studies, the members of type IA subclass are believed to effect DNA relaxation by a enzyme-bridged mechanism. ${ }^{9}$ However, unlike the vaccinia topoisomerase, E. coli topoisomerase I does not exhibit high degree of sequence specificity, and hence only limited information on interaction with substrate and reaction mechanism is available. In this manuscript, we have taken the advantage of site-specific binding of Mycobacterium smegmatis topoisomerase I to address the possible reaction mechanism of type 1A topoisomerases. M. smegmatis topoisomerase I has several unusual features. While the protein exhibits characteristics akin to type IA topoisomerases it lacks the zinc-finger motif, found in other members of type IA family. ${ }^{25}$ This is the only known topoisomerase that interacts with both single and double-stranded DNA with comparable affinity, ${ }^{26}$ and displays a high degree of sequence specificity. ${ }^{26,27}$ These features of topoisomerase I make it a suitable candidate to address the molecular basis of site-specific interaction. Here, we describe the interaction pattern of M. smegmatis topoisomerase I with DNA. The novel footprinting pattern differs from any other topoisomerase I characterized so far, and provides clues into the reaction mechanism.

\section{Results}

\section{M. smegmatis topoisomerase I interacts with DNA in a sequence specific manner}

Mycobacterial topoisomerase I displays remarkable sequence specificity in recognizing the DNA sites containing the CGCTTC/G element. ${ }^{26,27}$ A 38mer oligonucleotide harboring this strong topoisomerase site (STS) was used in all footprinting experiments (see Experimental Procedures).

Incubation of topoisomerase I with STS containing oligonucleotides resulted in a distinct topoisomerase-DNA complex (Figure 1(a), lanes 1 and 2 ). The absence of such a retarded complex upon incubating the protein with non-specific oligonucleotides under identical conditions (Figure 1(a), lane 4) demonstrates the specificity of the interaction of the enzyme to the DNA having recognition sequence. Similar results were obtained in topoisomerase-mediated cleavage reactions of single and double-stranded DNA (Figure 1(b), lanes 2 and 3). No cleavage product was detected when non-specific oligonucleotides were used (Figure 1(b), lane 4).

\section{DNase / footprinting}

DNase I footprinting assays provide overall protection pattern showing the surface area of DNA occupied by the protein. The end-labeled

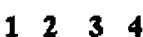

A

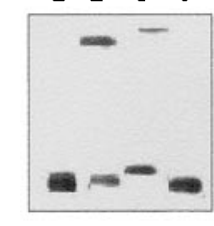

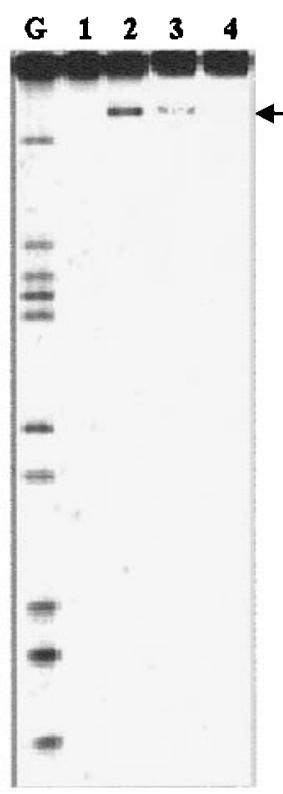

Figure 1. Characterization of the substrate DNA used in footprinting reaction. (a) Specific binding of topoisomerase I: oligonucleotides containing the STS (lanes 13) or lacking it (lane 4) were incubated either in the absence (lane 1) or in the presence of $1 \mathrm{ng}$ of $\mathrm{M}$. smegmatis topoisomerase I (lanes 2 and 3) and then electrophoresed through a native $8 \%$ polyacrylamide gel at $150 \mathrm{~V}$ for one hour. In lane 3, STS DNA in doublestrand context was used. (b) Specific cleavage of the STS oligonucleotide: G ladder (Lane G); free DNA (lane 1). Topoisomerase was incubated along with STS oligonucleotide $(10 \mathrm{fmol})$ in single (lane 2$)$ or double-stranded context (lane 3), or incubated with oligonucleotide lacking STS (lane 4). The reaction products were electrophoresed through a denaturing $16 \%$ polyacrylamide gel and exposed to autoradiography. The arrow indicates the topoisomerase cleavage site. 


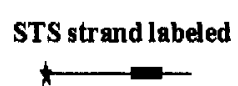

A

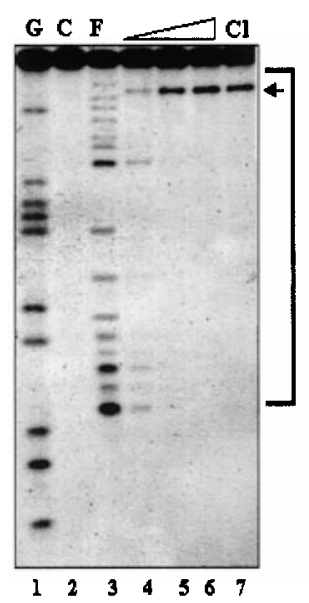

Non-STS str and labeled

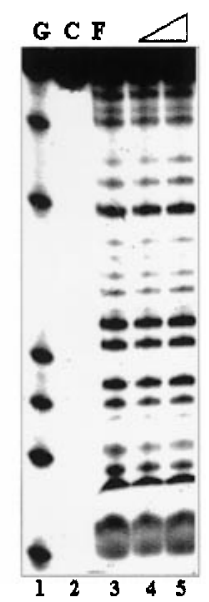

B

GAGAGGCGGTTTGCGTATT GGGCGCTCTTCCGCTTCCT-3' CTCTCCGCCAAACGCATAACCCGCGAGAAGGCGAAGGA-5

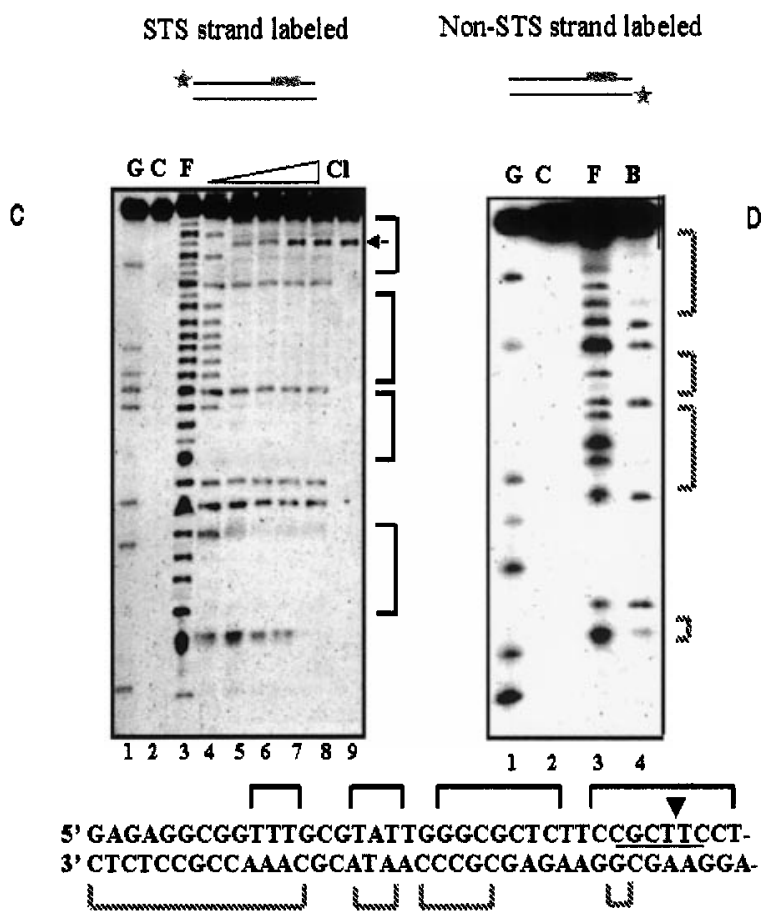

Figure 2. DNase I protection analysis. (a) Footprinting on single-stranded DNA 0.1 pmol of STS (represented by filled box) oligonucleotide was used in the assay. G ladder (lane 1); substrate DNA (C; lane 2), either treated with $5 \mathrm{ng}$ of DNase I alone (F; lane 3) or incubated with varying concentrations of topoisomerase I $(0.5,1$ and $5 \mathrm{ng}$ ) prior to the DNase I treatment (lanes 4-6, respectively). Topoisomerase I (10 ng) cleavage product obtained by incubating it with the substrate is indicated by an arrow (Cl; Lane 7). (b) Footprinting on non-STS oligonucleotides. G ladder (lane 1); labeled DNA (C; lane 2), was either incubated with DNase I alone (F; lane 3) or incubated with $5 \mathrm{ng}$ (lane 4) or $10 \mathrm{ng}$ (lane 5) of topoisomerase I and then treated with $5 \mathrm{ng}$ of DNase I. (c) Footprinting on double-stranded DNA. Labeled STS DNA in double-stranded context was used. G lad- substrate was incubated with topoisomerase and then treated with DNase I. Such an analysis with single-stranded DNA containing STS is shown in Figure 2(a). The nucleolytic probing of DNA-protein complex revealed that the bound protein protects the phosphodiester backbone of the entire 38mer DNA from DNase I catalyzed hydrolysis (Figure 2(a)). When the complementary strand lacking the STS, thus representing the non-specific sequence, was used instead, topoisomerase did not confer any protection (Figure 2(b)). Unlike in Figure 2(a), an interrupted protection pattern was obtained when double-stranded DNA (having the STS strand labeled) was subjected to footprinting analysis (Figure 2(c)). While cleavage of phosphodiester bonds between residues $-5 \mathrm{C}$ and $-6 \mathrm{~T},-13 \mathrm{G}$ and $-14 \mathrm{G},-19 \mathrm{~T}$ and $-20 \mathrm{G}$, and $-25 \mathrm{~T}$ and $-26 \mathrm{G}$ residues are largely unaltered in the unbound and bound states, other regions appear to be well protected by the enzyme. This pattern of protection is distinct from what is known for other topoisomerases. In both the single and double-stranded context the protein protects a region spanning about 30 bases. Although nonscissile strand by itself does not show any protection (Figure 2(b)), it is possible that some regions are protected in double-stranded context as mycobacterial topoisomerase I binds to double-stranded DNA. ${ }^{26,27}$ To address the point, the labeled bottom strand was annealed to the unlabeled top strand and used in the protection assay. In this case also the bottom strand was protected in double-stranded context (Figure 2(d)). The protection conferred by topoisomerase at the $5^{\prime}$ termini could not be clearly ascertained as DNase I poorly cleaves this region in oligonucleotides. $^{28}$

der (lane 1); STS DNA, untreated (C; lane 2), pre-incubated either in the absence (F; lane 3 ) or in the presence of 1, 2.5, 5, 10 and $15 \mathrm{ng}$ of topoisomerase I (lanes 4-8, respectively) and then treated with $5 \mathrm{ng}$ of DNase I. Topoisomerase I (15 ng) mediated cleavage reaction is indicated by an arrow ( $\mathrm{Cl}$; lane 9). (d) Labeled non-STS oligonucleotide in double-stranded context was used for the assay. G ladder (lane 1); the duplex DNA, untreated (C; lane 2), pre-incubated either in the absence ( $F$; lane $3)$ or in the presence (B; lane 4 ) of topoisomerase I (10 ng) and then subjected to DNase I digestion. In all the cases the reaction products were resolved through $16 \%$ urea-PAGE. Parenthesis marks the region of protection and is indicated in the sequence below. The sequence underlined represents the STS. 


\section{Base-specific recognition by mycobacterial topoisomerase I}

\section{Topoisomerase I contacts a $G$ residue within the STS}

One of the ways of target recognition by sitespecific DNA-binding proteins is by direct contact with the residues in the major and/or minor groove, enabling discrimination of specific sequence from the unrelated ones. In order to map the residues, which are in close proximity of the protein, DMS protection and interference assays were carried out. The reagent methylates the N-7 position of guanine bases in the major groove and $\mathrm{N}-3$ of adenine bases in the minor groove. ${ }^{29} \mathrm{Com}-$ parison of piperidine cleavage products following DMS treatment revealed protection of a guanine

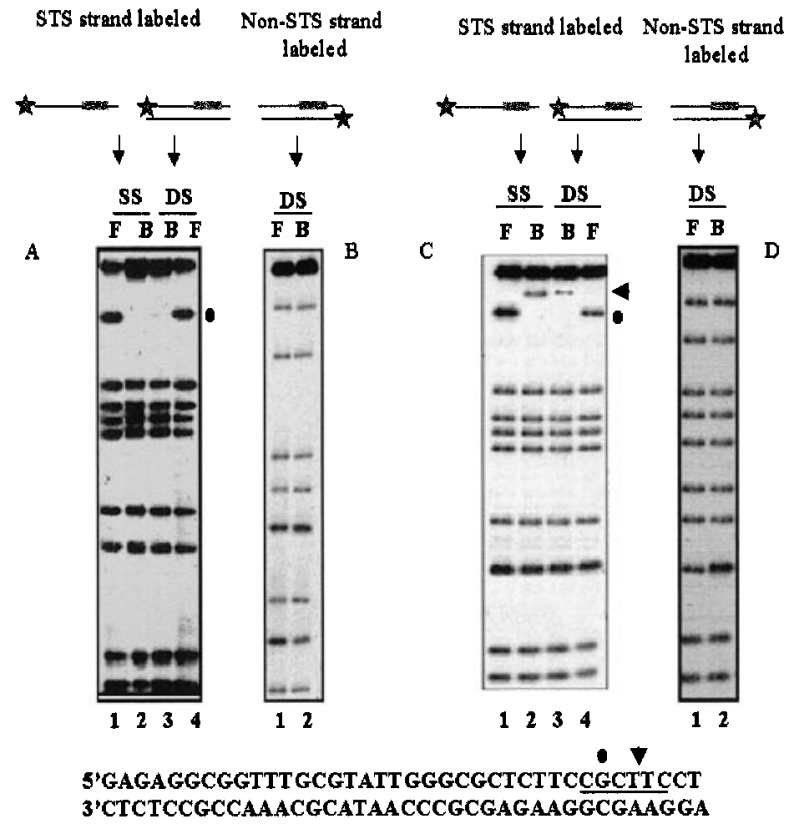

Figure 3. DMS reactions: methylation protection (a) Topoisomerase I (150 ng) was incubated with STS oligonucleotide in single (lane 2) or double-stranded context (lane 3) and subjected to DMS modification. SS indicates scissile strand in single-stranded context (lanes 1 and 2) while DS represents the double-strand context (lanes 3 and 4). (b) Labeled non-STS DNA was incubated with topoisomerase I (300 ng) in double-stranded (DS) context prior to methylation by DMS. Methylation interference (c) Labeled STS oligonucleotide was premethylated and incubated with topoisomerase I (150 ng). DNA corresponding to free (lane F) and DNAprotein complex (lane B) are indicated. Topoisomerase mediated cleavage is indicated by an arrow. (d) Labeled non-STS DNA was pre-methylated, hybridized to unlabeled STS strand and incubated with topoisomerase I (300 ng). In all the panels, F and B represent the free and bound fractions, respectively. Filled boxes represent the STS while the filled circles indicate the G contact. Topoisomerase induced cleavage is indicated by an arrow, while the STS sequence is underlined. residue present within the recognition sequence (Figure 3(a), lane 2). The results were identical in double-stranded context as well (Figure 3(a), lane 3). The single $G$ residue present in the minimal recognition site was protected (Figure 3(a) lanes 2 and 3 ). No additional $G$ residue contacts could be detected when duplex DNA having the label in non-scissile strand was used as a substrate (Figure 3(b)).

Binding of topoisomerase I was also assayed following methylation of the oligonucleotide substrate in single strand context. DNA corresponding to the free and bound fractions were separated and analyzed after piperidine cleavage. Comparison of the cleavage products revealed the near complete exclusion of the piperidine cleavable site corresponding to the $-3 G$ in the scissile strand from the bound fraction (Figure 3(c), lane 2). In contrast, methylation of $G$ residues, situated elsewhere did not affect the binding. The pattern of methylation interference was similar in double-stranded DNA (Figure 3(c), lane 3) to results obtained with single strand DNA (Figure 3(c), lane 2). A cleavage product of the radiolabeled scissile strand was detected in the protein-bound DNA fraction, but not in the free DNA population (Figure 3(c), lane B). The $\mathrm{G}$ residue protected (Figure 3(a)) from chemical modification was the same residue whose premodification interfered with DNA binding. No interactions were detected with the non-scissile strand in double-stranded context (Figure 3(d)).

\section{Additional base contacts of topoisomerase I revealed by missing contact experiments}

As the previous set of experiments revealed only a single base specific contact and DNase I footprinting showed a large protected region, we investigated whether there were any additional interactions. Moreover, a single hydrogen bond is unlikely to be the sole determinant of the sequence specific recognition. ${ }^{30}$ In addition, our previous studies with the STS-containing synthetic oligonucleotides having different base substituents revealed the importance of +1 and +2 residues. ${ }^{27}$ In order to identify the contribution of other nucleotides in high-specificity interaction of the topoisomerase I, missing nucleoside interference analyses were performed. ${ }^{31,32}$ Protein was incubated with the modified DNA and the bound fractions were separated as described in experimental procedures. Depurination of the $-3 G$ residue within the STS interfered with the topoisomerase I binding (Figure 4(a), lane 2). This is the same G residue shown to interact with enzyme by DMS protection and interference assays. In contrast, purines located at the immediate upstream of the minimal motif (CGCTTC/G; the recognition sequence for the site-specific binding of topoisomerase $\mathrm{I}^{26,27}$ ) do not seem to be in close proximity to the protein (Figure 4(a) lane 2). Similar results were obtained in double-stranded context 


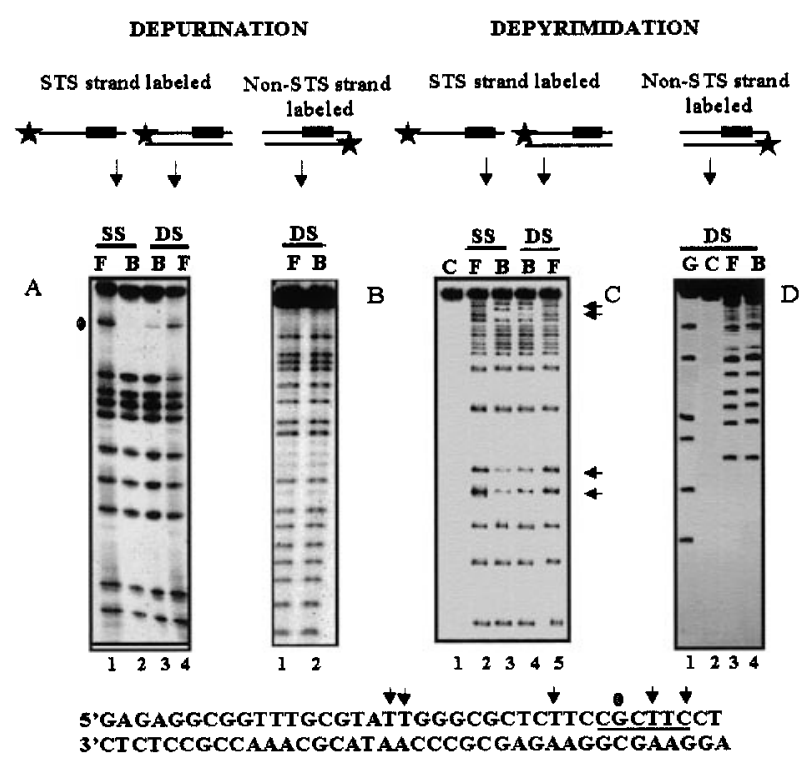

Figure 4. Missing base contacts experiments: depurination (a) The labeled scissile strand was modified using formic acid and incubated with $150 \mathrm{ng}$ of topoisomerase I. DNA corresponding to free (F; lane 1 ) and bound fractions (B; lane 2 ) are indicated. STS strand was depurinated, annealed to the unlabeled bottom strand and then incubated with $300 \mathrm{ng}$ of topoisomerase I. The bound (B; lane 3$)$ or free (F; lane 4$)$ fractions are indicated. (b) Labeled non-STS strand was depurinated, annealed to the unlabeled STS strand and then incubated with $300 \mathrm{ng}$ of topoisomerase I. The free $(\mathrm{F})$ and bound (B) fractions (lanes 1 and 2, respectively) are shown. Depyrimidation (c) The labeled STS strand (C; lane 1) was modified by hydrazine and incubated with $150 \mathrm{ng}$ of topoisomerase I (lanes 2 and 3) or annealed to non-STS strand (lanes 4 and 5) prior to incubation with $300 \mathrm{ng}$ of topoisomerase I. The free (F; lanes 2 and 5) and bound (B; lanes 3 and 4$)$ fractions are indicated. (d) Labeled non-STS DNA was depyrimidated, annealed to the unlabeled scissile strand and used for the assay. G ladder (lane 1); the substrate DNA (C; lane 2) was incubated with $300 \mathrm{ng}$ of topoisomerase I. The free (F; lane 3 ) and bound (B; lane 4) fractions indicated. The purine contact is represented by the filled circle while the arrows indicate the pyrimidine contacts. The sequence underlined represents the STS.

(Figure 4(a) lane 3). Purine residues in the bottom strand do not seem to be contacted by the protein (Figure 4(b)).

Depyrimidation experiments revealed the important pyrimidine contacts of the protein (Figure 4(c), lanes 2 and 3). These base contacts are localized in two distinct regions in the DNA. Two of these contacts were with $+2 \mathrm{C}$ and $-1 \mathrm{~T}$ within the STS whereas other critical contacts were with $-7 \mathrm{~T},-16 \mathrm{~T}$ and $-17 \mathrm{~T}$ residues, upstream to the STS. Pyrimidine-specific contacts were not observed with bottom strand when annealed to the scissile strand (Figure 4(d)).

\section{Electrostatic interactions with DNA backbone of the scissile strand}

Apart from the base-specific contacts, ionic interactions between the DNA and protein contribute towards the energy required for binding. In addition, sequence-specific binding is enhanced by DNA backbone contacts with the protein as they govern the geometry between protein and DNA. ${ }^{33}$ Phosphate contacts made by mycobacterial topoisomerase I were determined using ethylnitrosourea-modified substrate DNA. The footprinting pattern obtained is shown in Figure 5 depicting the comparison of the distribution of products in bound and free fractions. Each fragment moves as a doublet representing heterogeneous population of 3'-hydroxyl and $3^{\prime}$-ethylphosphates. ${ }^{32}$ As can be seen in Figure 5, the strongest inhibition to binding

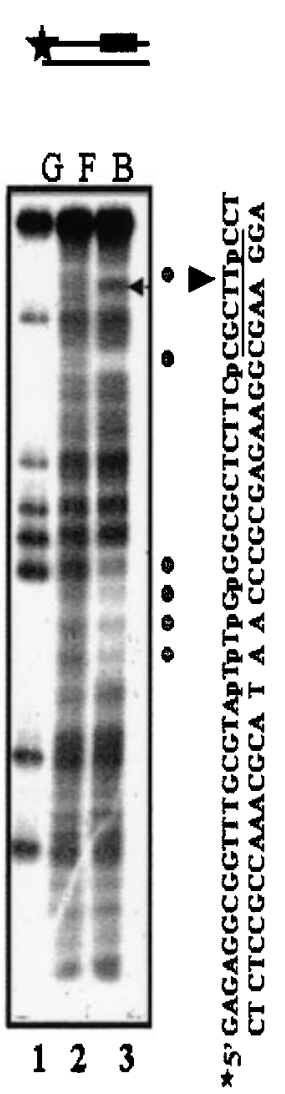

Figure 5. Phosphate contacts. STS DNA was modified by ethylnitrosourea, and incubated with $100 \mathrm{ng}$ topoisomerase I. The DNA-protein complex was separated from the free DNA on a native $8 \%$ polyacrylamide gel, purified and cleaved at the modified sites, as described in Experimental Procedures. G ladder (lane 1); free DNA (F; lane 2); bound fraction (B, lane 3). STS is marked by filled box. The phosphate contacts are indicated by filled circles and represented by " $p$ " in the sequence. Topoisomerase cleavage site is indicated by an arrow, while the STS is represented by the underlined sequence. 
occurs when the two phosphates (p) in the sequence TCpCGCTTpC are ethylated. The sites of complete interference therefore correspond to important contacts between the DNA and the protein. Ethylation at other sites inhibits the complex formation to varying degrees. When the contiguous phosphates upstream to the STS in the sequence 5'-TApTpTpGpG are modified, the binding is partly inhibited. Thus interaction with six sugar phosphates seems to be important for DNA binding, five of which are localized upstream to the recognition sequence, and only one is within the STS. No phosphate contacts are established with the bottom strand (not shown).

\section{Change in the B-DNA conformation of substrate DNA}

Although the enzyme also binds duplex DNA efficiently and protects it from nuclease digestion, base- and backbone-probing experiments revealed contacts only in the scissile strand. To better understand the interaction, chemical nuclease, 1,10 phenanthroline-copper, a redox-active coordination complex that nicks the phosphodiester backbone of DNA by oxidative degradation of the deoxyribose moiety, was used. Unlike the DNase I protection, $\mathrm{Cu}(\mathrm{OP})_{2}$ probing did not result in protection of the recognition sequence (Figure 6(a)). Similar observations were made with BspRI methyl transferase; while DNase I footprinting revealed a protected region, $\mathrm{Cu}(\mathrm{OP})_{2}$ failed to show any protection with BspRI. ${ }^{34}$ DNase I, interacts with both the major and minor groove, ${ }^{35}$ whereas the chemical nuclease is known to bind only to the minor groove. ${ }^{35}$ Thus, the results depicted in Figure 6(a) suggest that the protein either does not make any minor groove contact or even if it does, it does not alter the conformation of the minor groove so as to block the accessibility of 1,10 phenonthrolinecopper to hydrogen. A stretch of four residues at the positions -17 to -20 (Figure 6(a)), upstream to cleavage site revealed enhanced reactivity to $\mathrm{Cu}(\mathrm{OP})_{2}$ in spite of lack of intrinsic preference of the chemical nuclease for $\mathrm{A}$ and $\mathrm{T}$ residues. $^{36}$ The hyper-reactivity of these residues reflects protein-induced alteration of DNA conformation. Additional support for the distortion was obtained by conformational mapping of the substrates with $\mathrm{KMnO}_{4}$, which selectively modifies the unstacked thymidine residues. Since three of the four hyperactive residues observed in Figure 6(a) happen to be $\mathrm{T}$, it should be reactive to the $\mathrm{KMnO}_{4}$, provided these residues exist in unstacked conformation. Figure 6(b) demonstrates the structural deformity of DNA at these $T$ residues. A point to be noted is that two of these $\mathrm{T}$ residues were shown to be important for protein binding, as revealed by missing nucleoside experiments (see Figure 4(c)).

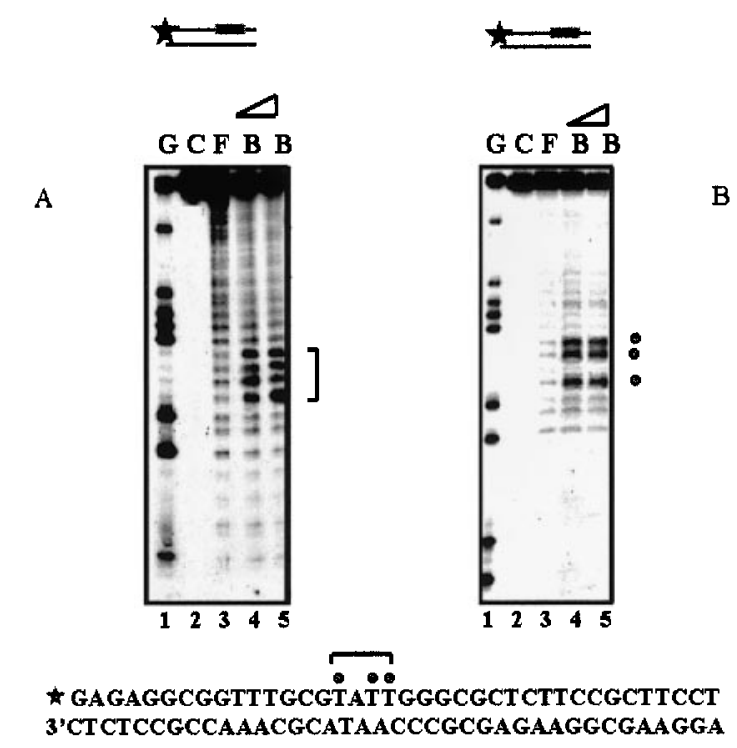

Figure 6. Structural distortion in DNA. STS oligonucleotide annealed to the non-STS DNA was used for the assays. The reaction products were resolved on a denaturing $18 \%$ polyacrylamide gel and autoradiographed. (a) $\mathrm{Cu}(\mathrm{OP})_{2}$ footprinting. $\mathrm{G}$ ladder (lane 1), substrate DNA (C; lane 2), was treated with $\mathrm{Cu}(\mathrm{OP})_{2}$ in the absence (F; lane 3) or presence of 100 and $200 \mathrm{ng}$ of topoisomerase I (B; lanes 4 and 5, respectively). Parenthesis indicates the hypersites. (b) $\mathrm{KMnO}_{4}$ footprinting. $\mathrm{G}$ ladder (lane 1); substrate DNA (C; lane 2), incubated either in the absence (F; lane 3$)$ or in the presence of 50 (B; lane 4) and $100 \mathrm{ng}(\mathrm{B}$; lane 5$)$ of topoisomerase and then probed with $\mathrm{KMnO}_{4}$. Filled circles correspond to the hyper-reactive residues and are indicated in the sequence below.

\section{Discussion}

Footprinting analyses probing the M. smegmatis topoisomerase I-DNA complex, enabled us to determine the different types of contacts responsible for specific interactions. The recognition pattern of the enzyme has novel features not seen so far for any other topoisomerase, providing insights into the reaction mechanism. From the overall interaction pattern it appears that the enzyme employs an enzyme-bridged mechanism to remove negative supercoils from DNA.

\section{DNA binding of mycobacterial topoisomerase I}

The DNase I footprint of mycobacterial topoisomerase I spans a region of about 30 bases in both single and double-stranded context suggesting a large surface area of interaction. In contrast, E. coli topoisomerase III, which is known to show some sequence specificity protects a region of 14 nucleotides in single-stranded DNA. ${ }^{37}$ However, other members of 1A group, E. coli and Micrococcal luteus topoisomerase I, do not form 
clear footprints, and are shown to protect a region of more than 50 nucleotides on both the strands of DNA, indicating the binding of several topoisomerase molecules. ${ }^{38}$ It is noteworthy that both E. coli and $M$. luteus enzymes do not exhibit remarkable sequence specificity unlike the former two enzymes.

The base-specific and phosphate contacts of mycobacterial topoisomerase is bipartite and restricted to the scissile strand (Figure 7). The six hydrogen-mediated base contacts established by the protein involves a lone purine contact at $-3 \mathrm{G}$ and five pyrimidines $+2 \mathrm{C},-1 \mathrm{~T},-7 \mathrm{~T},-16 \mathrm{~T}$ and $-17 \mathrm{~T}$. Three of these base-specific interactions are within the STS, namely, $+2 \mathrm{C},-1 \mathrm{~T}$ and $-3 \mathrm{G}$. From the phosphate interference assay it appears that six electrostatic contacts are important for proteinDNA interaction, one of which is within the STS between the $+1 \mathrm{~T}$ and $+2 \mathrm{C}$ (Figure 7). Though interactions of mycobacterial topoisomerase I map on both the faces of the helix, the protein contacts only the top strand (Figure 7). Thus, mycobacterial topoisomerase is intrinsically a single-strand DNAbinding protein, with the ability to recognize the scissile strand in duplex context, as well with a marginally reduced affinity (Figures 1(b), 3(a), 4(a)). The E. coli MutL protein involved in DNA repair exhibits similar binding features wherein affinity for single-stranded DNA is twofold higher than for double-stranded DNA. ${ }^{39}$ These results are expected as single-stranded DNA is susceptible to a variety of interactions between the phosphate backbone, surrounding water molecules and the cations, it can adopt several conformations. In contrast, determinants that shape the helical geometry, i.e. the stacking interactions and the inter-phosphate repulsion, ${ }^{40,41}$ impose restrictions on the movement of the sugar phosphate backbone, which in turn affect the flexibility of the bases in the double-stranded context. ${ }^{42}$ Additionally, the functional groups of the bases in double-stranded DNA are less accessible owing to a constellation of

A

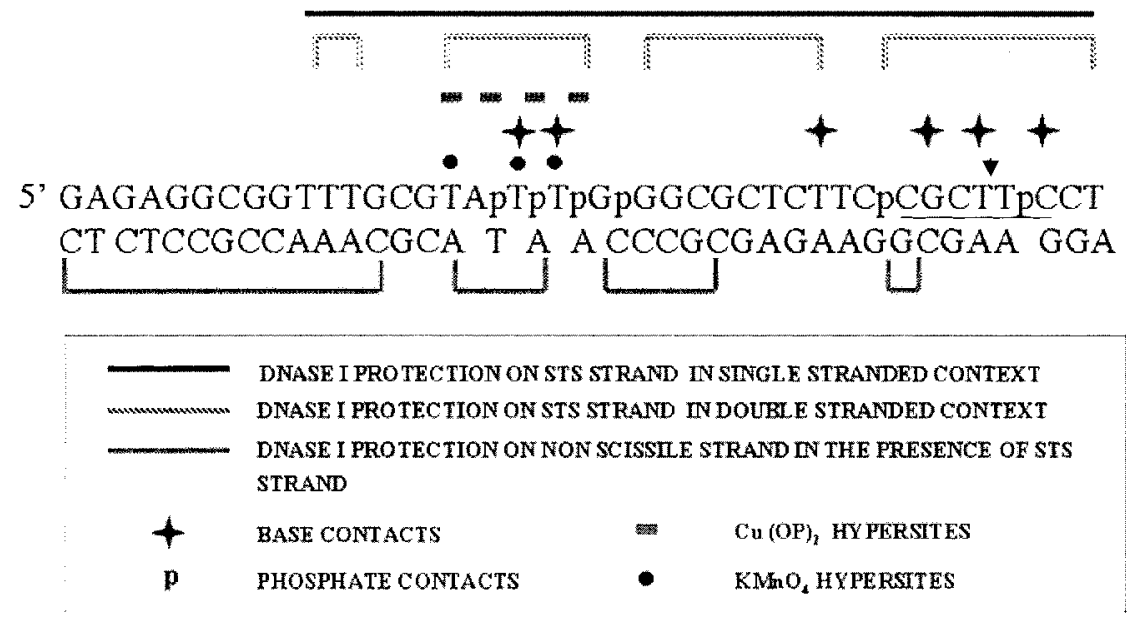

B

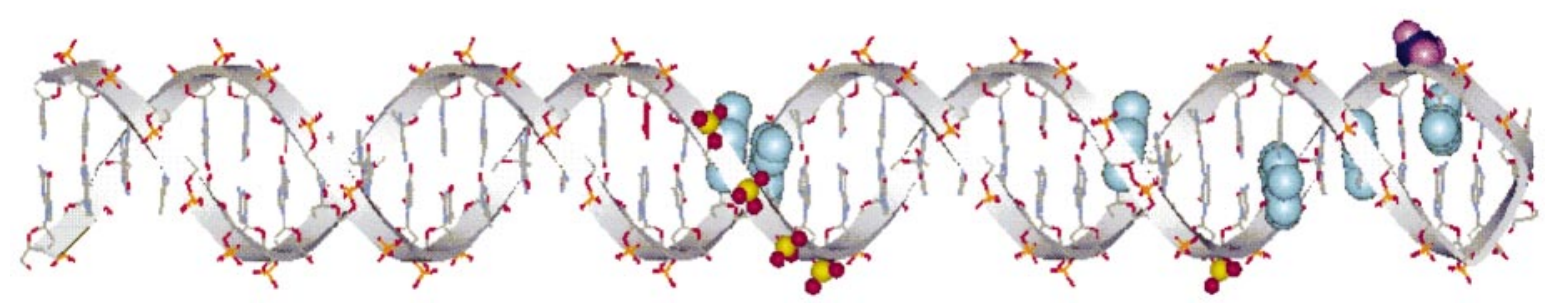

Figure 7. (a) Summary of Footprinting data. The sequence underlined represents the STS and the arrow indicates the cleavage site. (b) Modeling of the substrate DNA: The 38mer substrate DNA was modeled using Molscript and visualized in Weblab viewer Lite 3.5. Base specific contacts (in cyan) and phosphate contacts (in yellow) are shown. The scissile phosphate is indicated (in blue). 
atoms from sugar and the complementary bases. Interactions between the charged, as well as uncharged molecules, and the van der Waals repulsion from the neighboring atoms both in plane and out of plane could restrict free ligand binding. The higher degree of flexibility and easier accessibility of the functional groups in singlestranded DNA could attribute to the marginal differences in the contact pattern of the enzyme in single and double-stranded context.

\section{Structural distortion of DNA}

Although the DNA determinants of enzyme contact lie in the scissile strand, the enzyme exhibits a remarkable ability to interact with double-stranded DNA. This possibly stems from the property of the enzyme to distort double-stranded DNA after binding. The mycobacterial topoisomerase I induces localized structural distortion upstream to STS. $\mathrm{KMnO}_{4}$ probing suggests the formation of single-strand region at the T-T-A-T stretch (17 to $-20)$, which exhibits hypersensitive cleavage. The strong hold on the scissile strand by the enzyme within the same sequence is reflected by the basespecific contacts with two of these residues apart from the four phosphate contacts. Thus, region upstream to the cleavage site, seems to play a crucial role in defining the geometry of the proteinDNA interface, and may enhance the sequencespecific binding. Phosphate contacts, as well as conformational change induced by protein-DNA phosphate contacts, have been implicated to aid the process of sequence recognition. ${ }^{33}$

\section{Modeling the DNA}

Summary of different footprinting results presented in Figure 7(a) is projected as a molecular model of the DNA contact sites of M. smegmatis topoisomerase I (Figure 7(b)). Analysis of the DNA contacts by the enzyme in the context of B-DNA model, reveals important features. The protein interacts with its substrate DNA on both the sides of double helix as the protein-base and proteinphosphate contacts are arrayed across the double helix. This mode of interaction is reminiscent of the DNA interaction pattern of topoisomerases wherein the protein clamps the DNA within the centrally placed core..$^{9,43}$ Based on the pattern, it is postulated that the mycobacterial topoisomerase I wraps around the DNA duplex.

\section{DNA relaxation mechanism of mycobacterial topoisomerase I}

Two models have been proposed to explain the DNA relaxation mechanism catalyzed by topoisomerase I. The "free rotation model" proposes that the scissile strand is free to swivel about the intact strand as the enzyme does not directly contact the DNA downstream to cleavage site. The model implies multiple relaxation events per round of cleavage-religation cycle. According to the second model known as "strand passage model" (or "enzyme-bridged model"), the enzyme regulates the relaxation cycle by maintaining a strong hold on the DNA upstream and downstream to the nick. A single relaxation event is permitted per cycle of cleavage-religation reaction. Studies support the former mechanism to be operating in the case of vaccinia topoisomerase $\mathrm{I}^{22}$ and strand passage model for type IA topoisomerases. ${ }^{9}$ The mycobacterial topoisomerase I, interacts with the upstream, as well as downstream sequences with respect to nick (Figure 7). In addition to covalent contact with +1 site, both base-specific $(+2)$ and phosphate contacts indicate tight grip of the enzyme downstream to the cleavage site. Yet the enzyme has another stranglehold as a number of base-specific and phosphate contacts are located at a distance upstream to the cleavage site. The results are consistent with the strand passage model wherein the enzyme holds both the sides of the nick to prevent free axial rotation about the non-cleaved strand for M. smegmatis enzyme function.

In summary, the protein-DNA contacts mapped provide the basis for site-specificity demonstrated by mycobacterial topoisomerase I. The present analyses illustrate novel features of a type IA topoisomerase interaction with DNA. Solution structure studies in conjunction with mutational studies would aid in further delineating the relaxation mechanism employed by $M$. smegmatis topoisomerase $\mathrm{I}$.

\section{Experimental procedures}

\section{Purification of topoisomerase I}

Topoisomerase I was purified from wild-type M. smegmatis SN2 cells, as described. ${ }^{25,44}$ The amount of enzyme catalyzing $50 \%$ relaxation of $300 \mathrm{ng}$ of supercoiled plasmid at $37^{\circ} \mathrm{C}, 30$ minutes in a reaction volume of $20 \mu \mathrm{l}$ is defined as 1 unit, and corresponds to $2 \mathrm{ng}$ of the purified protein.

\section{Oligonucleotide substrates}

Oligonucleotide (38 mer) containing a strong topoisomerase site (STS oligonucleotide; 5'-GAG AGGCGGTTTGCGTATTGGGCGCTCTTCCGCTTCCT) was used as the specific substrate in all the assays. The complementary oligonucleotide (non-STS oligonucleotide; 5'-AGGAAGCGGAAGAGCGCCCAATACGCAAA CCG CCTCTC) served as the non-specific substrate. When double-stranded oligonucleotides were used, 2.5fold molar excess of unlabeled complementary strand was annealed to the labeled strand by incubating at $95^{\circ} \mathrm{C}$ for five minutes followed by slow cooling to room temperature. Double-strandedness of DNA was confirmed by electrophoresing an aliquot of the reaction mix through $10 \%(\mathrm{w} / \mathrm{v})$ non-denaturing polyacrylamide gel. Oligonucleotides were $5^{\prime}$ end-labeled using bacteriophage T4 polynucleotide kinase and $\left[\gamma-\mathrm{P}^{32}\right]$ ATP $(6000$ 
$\mathrm{Ci} / \mathrm{mmol}$ ) and purified using G-50 spin column chromatography.

\section{Binding and cleavage reactions}

The assays for non-covalent enzyme DNA complexes and cleavage reactions were performed, as described. ${ }^{27}$

\section{DNase I footprinting}

Reaction mixtures $(20 \mu \mathrm{l})$ containing $40 \mathrm{mM}$ Tris- $\mathrm{HCl}$ (pH 8.0), $20 \mathrm{mM} \mathrm{NaCl}, 1 \mathrm{mM} \mathrm{Mg}{ }^{2+}, 0.5 \mathrm{mM} \mathrm{Ca}^{2+}$ and $100 \mathrm{fmol}$ of radiolabeled oligonucleotides were incubated on ice for five minutes along with topoisomerase I. The samples were thereafter treated with $5 \mathrm{ng}$ of DNase I (Worthington Biochemical Corp.) for 30 seconds at $24^{\circ} \mathrm{C}$. The reactions were terminated by the addition of stop buffer (50 mM Tris- $\mathrm{HCl}(\mathrm{pH} 8.0), 150 \mathrm{mM} \mathrm{NaCl}$ and $10 \mathrm{mM}$ EDTA). The products of the reactions were autoradiographed following separation using $16 \%$ denaturing PAGE at $2000 \mathrm{~V}$ for two hours.

\section{Methylation protection and interference assay}

Topoisomerase I (150 ng or $300 \mathrm{ng}$ ) was incubated with the end-labeled oligonucleotides $(200,000 \mathrm{cpm})$ in binding buffer (40 mM Tris- $\mathrm{HCl}(\mathrm{pH} \quad 8.0), 20 \mathrm{mM}$ $\mathrm{NaCl})$ in $10 \mu \mathrm{l}$ reaction volume. After increasing the volume to $50 \mu \mathrm{l}$ the reaction mix was treated with $1 \%$ DMS and vortexed vigorously for 1.5 minutes. The reaction was terminated by the addition of $1.5 \mathrm{M}$ sodium acetate and $1 \mathrm{M} \beta$-mercaptoethanol. Following precipitation, DNA was re-suspended in $90 \mu \mathrm{l}$ of $\mathrm{H}_{2} \mathrm{O}$ and $10 \mu \mathrm{l}$ of piperidine. The samples were thereafter incubated at $90^{\circ} \mathrm{C}$ for 30 minutes, vacuum-dried and suspended in $6 \mu \mathrm{l}$ of formamide dye. Control reactions containing radiolabeled DNA without added enzyme were processed in identical fashion. Cleavage products were analyzed following separation through a denaturing $18 \%$ polyacrylamide gel at $2000 \mathrm{~V}$ for two hours. For interference assay, the oligonucleotide substrates were pre-methylated by treating with $1 \%$ DMS for one minute at room temperature. Modification was stopped by the addition of $50 \mu 1$ of DMS stop buffer (1.5 $\mathrm{M}$ sodium acetate, $1 \mathrm{M} \beta$-mercaptoethanol). DNA was precipitated and suspended in water. Binding reactions were performed, as described in methylation protection assay. DNA corresponding to free and the bound fractions were separated by electrophoresing through a native $8 \%$ polyacrylamide gel. After autoradiography the corresponding regions were excised and eluted. The samples were purified using NAP-5 columns, vacuum dried and re-suspended in $100 \mu \mathrm{l}$ of binding buffer. After the cleavage by piperidine the samples were electrophoresed, as described in previous section.

\section{Depurination and depyrimidation reaction}

Purines and the pyrimidine specific reactions were performed as described earlier. ${ }^{31}$ Oligonucleotides were annealed to the complementary, unmodified strand when duplex DNA was used. Binding reactions were performed as described above. The reaction products were resolved through a denaturing $18 \%$ polyacrylamide gel.

\section{Ethylation interference assay}

Saturated solution of ethyl-nitrosourea (EtNU) in ethanol was added to end-labeled oligonucleotides $(200,000 \mathrm{cpm})$ and incubated with $100 \mu \mathrm{l}$ of $50 \mathrm{mM}$ cacodylate buffer $(\mathrm{pH} 8.0)$ at $50^{\circ} \mathrm{C}$ for 30 minutes. DNA was ethanol precipitated, and suspended in $50 \mu \mathrm{l}$ of water. Binding reactions and isolation of free and bound forms of DNA were as described above. Five microliters of $1 \mathrm{M} \mathrm{NaOH}$ was added to the samples and incubated at $90^{\circ} \mathrm{C}$ for 30 minutes and then neutralized with $\mathrm{HCl}$. DNA was precipitated and suspended in $5 \mu \mathrm{l}$ of formamide dye and electrophoresed through a urea $16 \%$ polyacrylamide gel. The gel was then exposed for autoradiography.

\section{$\mathrm{Cu}(\mathrm{OP})_{2}$ footprinting}

Footprinting reactions were performed as described earlier. $^{35,45}$ Briefly, topoisomerase I (100-200 ng) was incubated with 1 pmol end-labeled DNA $\left(10^{5} \mathrm{cpm}\right)$ in $170 \mu \mathrm{l}$ of reaction buffer containing, $20 \mathrm{mM}$ Tris- $\mathrm{HCl}$ $(\mathrm{pH} \mathrm{8.0)}$ and $20 \mathrm{mM} \mathrm{NaCl}$ for five minutes on ice. The reaction was initiated by the addition of $4 \mathrm{mM} \mathrm{1,10-}$ phenanthroline, $0.3 \mathrm{mM} \mathrm{CuSO}_{4}$ and $58 \mathrm{mM} 3$-mercaptopropionic acid and quenched after one minute at $25^{\circ} \mathrm{C}$, by the addition of $5 \mu \mathrm{l}$ of $100 \mathrm{mM}$ 2,9-dimethyl-1,10-phenanthroline. Following phenol-chloroform extractions DNA was precipitated, purified and the products were electrophoresed through a denaturing $18 \%$ polyacrylamide gel.

\section{$\mathrm{KMnO}_{4}$ footprinting}

Preformed DNA-protein complex was incubated with $2 \mathrm{mM} \mathrm{KMnO}_{4}$ for one minute at $25^{\circ} \mathrm{C}^{46}$ The reaction was stopped with $40 \mathrm{mM} \beta$-mercaptoethanol and $0.3 \mathrm{M}$ sodium acetate. Following ethanol precipitation and piperidine cleavage, the reaction products were electrophoresed through a denaturing $18 \%$ polyacrylamide gel.

\section{Acknowledgments}

We thank other members of the VN Lab and Kiran Bachhawat-Sikder for discussions. D.S. was supported by a project assistantship from Jawaharlal Nehru Centre for Advanced Scientific Research. The work is supported by a grant from Department of Biotechnology, Government of India.

\section{References}

1. Wang, J. C. (1996). DNA topoisomerases. Annu. Rev. Biochem. 65, 635-693.

2. Wang, J. C. (1971). Interaction between DNA and an Escherichia coli protein omega. J. Mol. Biol. 55, 523533.

3. Brown, P. O. \& Cozzarelli, N. R. (1979). A sign inversion mechanism for enzymatic supercoiling of DNA. Science, 206, 1081-1083.

4. Berger, J. M. (1998). Structure of DNA topoisomerases. Biochim. Biophys. Acta, 1400, 3-18.

5. Meima, R., Haan, G. J., Venema, G., Bron, S. \& de Jong, S. (1998). Sequence specificity of illegitimate plasmid recombination in Bacillus subtilis: possible 
recognition sites for DNA topoisomerase I. Nucl. Acids Res. 26, 2366-2373.

6. Kirkegaard, K., Pflugfelder, G. \& Wang, J. C. (1984). The cleavage of DNA by type-I DNA topoisomerases. Cold Spring Harbor Symp. Quant. Biol. 49, 411419.

7. Been, M. D. \& Champoux, J. J. (1984). Breakage of single-stranded DNA by eukaryotic type 1 topoisomerase occurs only at regions with the potential for base-pairing. J. Mol. Biol. 180, 515-531.

8. Shuman, S. \& Prescot, J. (1990). Specific DNA cleavage and binding by vaccinia virus DNA topoisomerase I. J. Biol. Chem. 265, 17826-17836.

9. Lima, C. D., Wang, J. C. \& Mondragon, A. (1994). Three-dimensional structure of the $67 \mathrm{~K} \mathrm{~N}$-terminal fragment of E. coli DNA topoisomerase I. Nature, 367, 138-146.

10. Yu, L., Zhu, C. X., Tse-Dinh, Y. C. \& Fesik, S. W. (1995). Solution structure of the C-terminal singlestranded DNA-binding domain of Escherichia coli topoisomerase I. Biochemistry, 34, 7622-7628.

11. Zhu, C.-X., Roche, C. J. \& Tse-Dinh, Y. C. (1997). Effect of $\mathrm{Mg}$ (II) binding on the structure and activity of Escherichia coli DNA topoisomerase I. J. Biol. Chem. 272, 16206-16210.

12. Morrison, A. \& Cozzarelli, N. R. (1979). Site-specific cleavage of DNA by E. coli DNA gyrase. Cell, 17, 180-184.

13. Edwards, K., Halligan, B., Davis, J., Nivera, N. \& Liu, L. (1982). Recognition sites of eukaryotic DNA topoisomerase I: DNA nucleotide sequencing analysis of topo I cleavage sites on SV40 DNA. Nucl. Acids Res. 10, 2565-2576.

14. Liu, L. F., Rowe, T. C., Yang, L., Tewey, K. \& Chen, G. L. (1983). Cleavage of DNA by mammalian DNA topoisomerase II. J. Biol. Chem. 258, 15365-15370.

15. Tse-Dinh, Y.-C., McCarron, B. G. H., Arentzen, R. \& Chowdhry, V. (1983). Mechanistic study of E. coli DNA topoisomerase I: cleavage of oligonucleotides. Nucl. Acids Res. 11, 8691-8701.

16. Bonven, B. J., Gocke, E. \& Westergaard, O. (1985). A high affinity topoisomerase I binding sequence is clustered at DNAase I hypersensitive sites in Tetrahymena R-chromatin. Cell, 41, 541-551.

17. Dean, F. B. \& Cozzarelli, N. R. (1985). Mechanism of strand passage by Escherichia coli topoisomerase I. The role of the required nick in catenation and knotting of duplex DNA. J. Biol. Chem. 260, 4984-4994.

18. Kovalosky, O. I., Kazyavkin, S. A. \& Slesarev, A. I. (1990). Archaebacterial reverse gyrase cleavage-site specificity is similar to that of eubacterial DNA topoisomerases I. Nucl. Acids Res. 18, 2801-2805.

19. Shuman, S. (1998). Vaccinia virus DNA topoisomerase: a model eukaryotic type IB enzyme. Biochim. Biophys. Acta, 1400, 321-337.

20. Sharma, A., Hanai, R. \& Mondragon, A. (1994). Crystal structure of the amino-terminal fragment of vaccinia virus DNA topoisomerase I at 1. $6 \AA$ resolution. Structure, 2, 767-771.

21. Cheng, C., Kussie, P., Pavletich, N. \& Shuman, S. (1998). Conservation of structure and mechanism between eukaryotic topoisomerase I and site-specific recombinases. Cell, 92, 841-850.

22. Stivers, J. T., Shuman, S. \& Mildvan, A. S. (1994). Vaccinia DNA topoisomerase I: kinetic evidence for general acid-base catalysis and a conformational step. Biochemistry, 33, 15449-15458.

23. Redinbo, M. R., Stewart, L., Kuhn, P., Champoux, J. J. \& Hol, W. G. (1998). Crystal structures of human topoisomerase I in covalent and noncovalent complexes with DNA. Science, 279, 1504-1513.

24. Stewart, L., Redinbo, M. R., Qiu, X., Hol, W. G. \& Champoux, J. J. (1998). A model for the mechanism of human topoisomerase I. Science, 279, 1534-1541.

25. Bhaduri, T., Bagui, T. K., Sikder, D. \& Nagaraja, V. (1998). DNA topoisomerase I from Mycobacterium smegmatis. An enzyme with distinct features. J. Biol. Chem. 273, 13925-13932.

26. Bhaduri, T., Sikder, D. \& Nagaraja, V. (1998). Sequence specific interaction of Mycobacterium smegmatis topoisomerase I with duplex DNA. Nucl. Acids Res. 26, 1668-1674.

27. Sikder, D. \& Nagaraja, V. (2000). Determination of the recognition sequence of Mycobacterium smegmatis topoisomerase I on mycobacterial genomic sequences. Nucl. Acids Res. 28, 1830-1837.

28. Drew, H. R. (1984). Structural specificities of five commonly used DNA nucleases. J. Mol. Biol. 176, 535-557.

29. Maxam, A. M. \& Gilbert, W. (1980). Sequencing end-labeled DNA with base-specific cleavages. Methods Enzymol. 65, 497-560.

30. Seeman, N. C., Rosenberg, J. M. \& Rich, A. (1976). Sequence-specific recognition of double helical nucleic acids by proteins. Proc. Natl Acad. Sci. USA, 73, 804-808.

31. Brunelle, A. \& Schleif, R. F. (1987). Missing contact probing of DNA-protein interactions. Proc. Natl Acad. Sci. USA, 84, 6673-6676.

32. Papp, P. P. \& Chattoraj, D. K. (1994). Missingbase and ethylation interference footprinting of P1 plasmid replication initiator. Nucl. Acids Res. 22, 152-157.

33. Pabo, C. O. \& Sauer, R. T. (1992). Transcription factors: structural families and principles of DNA recognition. Annu. Rev. Biochem. 61, 1053-1095.

34. Finta, C. \& Kiss, A. (1997). Footprint analysis of the bsp RI DNA methyltransferase-DNA interaction. Nucl. Acids Res. 25, 2841-2846.

35. Sigman, D. S., Spassky, A., Rimsky, S. \& Buc, H. (1985). Conformational analysis of lac promoters using the nuclease activity of 1,10-phenanthrolinecopper ion. Biopolymers, 24, 183-197.

36. Yoon, C., Kuawabara, M. D., Law, R., Wall, R. \& Sigman, D. S. (1988). Sequence-dependent variability of DNA structure. Influence of flanking sequences and fragment length on digestion by conformationally sensitive nucleases. J. Biol. Chem, 263, 8458-8463.

37. Zhang, H. L., Malpure, S. \& DiGate, R. J. (1995). Escherichia coli DNA topoisomerase III is a sitespecific DNA binding protein that binds asymmetrically to its cleavage site. J. Biol. Chem. 270, 2370023705.

38. Kirkegaard, K. \& Wang, J. C. (1985). Bacterial DNA topoisomerase I can relax positively supercoiled DNA containing a single-stranded loop. J. Mol. Biol. 185, 625-637.

39. Bende, S. M. \& Grafstrom, R. H. (1991). The DNA binding properties of the MutL protein isolated from Escherichia coli. Nucl. Acids Res. 19, 1549-1555.

40. Manning, G. S. (1978). The molecular theory of polyelectrolyte solutions with applications to the electrostatic properties of polynucleotides. Quart. Rev. Biophys. 11, 179-246.

41. Mirzabekov, A. D. \& Rich, A. (1979). Asymmetric lateral distribution of unshielded phosphate groups in nucleosomal DNA and its role in DNA bending. Proc. Natl Acad. Sci. USA, 76, 1118-1121. 
42. Hagerman, K. R. \& Hagerman, P. J. (1996). Helix rigidity of DNA: the meroduplex as an experimental paradigm. J. Mol. Biol. 260, 207-223.

43. Mondragon, A. \& DiGate, R. (1999). The structure of Escherichia coli DNA topoisomerase III. Struct. Fold Des. 7, 1373-1383.

44. Bhaduri, T. \& Nagaraja, V. (1994). DNA topoisomerase I from Mycobacterium smegmatis. Indian J. Biochem. Biophys. 31, 339-343.
45. Ramesh, V. \& Nagaraja, V. (1996). Sequencespecific DNA binding of the phage $\mathrm{Mu} C$ protein: footprinting analysis reveals altered DNA conformation upon protein binding. J. Mol. Biol. 260, 22-33.

46. Sandaltzopoulos, R. \& Papavassiliou, A. G. (1995). Use of potassium permanganate in in vitro footprinting analysis. Methods Mol. Cell. Biol. 5, 131134.

Edited by J. Karn 